\title{
Modularity and robustness of bone networks
}

\section{Matheus Palhares Viana, ${ }^{a}$ Esther Tanck, ${ }^{* b}$ Marcelo Emílio Beletti ${ }^{* c}$ and Luciano da Fontoura Costa $* a$}

\author{
Received 14th August 2008, Accepted 11th November 2008 \\ First published as an Advance Article on the web 12th January 2009 \\ DOI: $10.1039 / \mathbf{b 8 1 4 1 8 8 f}$
}

Cortical bones, essential for mechanical support and structure in many animals, involve a large number of canals organized in intricate fashion. By using state-of-the art image analysis and computer graphics, the 3D reconstruction of a whole bone (phalange) of a young chicken was obtained and represented in terms of a complex network where each canal was associated to an edge and every confluence of three or more canals yielded a respective node. The representation of the bone canal structure as a complex network has allowed several methods to be applied in order to characterize and analyze the canal system organization and the robustness. First, the distribution of the node degrees (i.e. the number of canals connected to each node) confirmed previous indications that bone canal networks follow a power law, and therefore present some highly connected nodes (hubs). The bone network was also found to be partitioned into communities or modules, i.e. groups of nodes which are more intensely connected to one another than with the rest of the network. We verified that each community exhibited distinct topological properties that are possibly linked with their specific function. In order to better understand the organization of the bone network, its resilience to two types of failures (random attack and cascaded failures) was also quantified comparatively to randomized and regular counterparts. The results indicate that the modular structure improves the robustness of the bone network when compared to a regular network with the same average degree and number of nodes. The effects of disease processes (e.g., osteoporosis) and mutations in genes (e.g., BMP4) that occur at the molecular level can now be investigated at the mesoscopic level by using network based approaches.

\section{Introduction}

The functionality of several biological systems is closely related to the respective geometry and spatial structure. For instance, the docking of proteins is strongly related to their geometrical shapes. At a more macroscopic scale, the shape of wings becomes critical for effective flight. To a great extent, the miracle of life strongly depends on the effective integration between shape and function not only along the life of individuals, but throughout the whole evolutionary chain. The anatomy of vertebrate cortical bones is no exception to the shape/function paradigm. Here, an intricate network of canals is used to convey the neurovascular system ${ }^{1,2}$ so as to remove toxic substances from the bone interior while nourishing the bone cells. At the same time, the organization of the canals is continuously modified in order to enhance the mechanical strength of the bone structure to the imposed solicitations. ${ }^{2,3}$ Differently from the others branched structures (typically trees) present in the human body, including the vessels

${ }^{a}$ Institute of Physics of São Carlos, University of São Paulo, São Carlos, Brazil.E-mail: ldfcosta@yahoo.com;

Fax: + 55163373 9879; Tel: + 551633739858

${ }^{b}$ Orthopaedic Research Lab, Radboud University Nijmegen Medical Center, Nijmegen, The Netherlands. E-mail: e.tanck@orthp.azn.nl; Tel: + 31243616959

${ }^{c}$ Institute of Medical Sciences, Federal University of Uberlândia, Uberlândia,Brazil.E-mail: mebeletti@pq.cnpq.br;

Fax: + 55343218 2430; Tel: +553432182240 network of the brain and the vascular and the system that conveys oxygen into lungs, the canal network of cortical bone exhibit a particularly intricate connectivity which includes many cycles. ${ }^{1}$

The new area of complex networks ${ }^{4-8}$ has provided the means to represent, analyze and model a large number of biological structures and systems, including protein-protein interaction, ${ }^{9,10}$ metabolism, ${ }^{11,12}$ and neuronal networks. ${ }^{13,14}$ Basically, complex networks are graphs (i.e. discrete structures composed by nodes interconnected through links or edges) that exhibit particularly intricate organization. It has been shown recently ${ }^{15}$ that, by associating each canal segment of the bone network to a link and every confluence of three or more canals to a respective node, the bone canal networks can be effectively represented and studied in terms of geographical complex networks, which consider not only the connectivity between nodes, but also their spatial positions. In addition to providing a precise representation of the connectivity of the bone canals, the transformation of the canal system into a network allows all the rich concepts and methods developed for complex networks to be immediately applied in order to investigate and identify several important structural features related to canal interconnectivity.

The bone network investigated in the present work was obtained by using a methodology similar to that applied in a previous work, ${ }^{15}$ where we showed that the bone network obtained from a portion of a cat femur had a degree 
distribution (i.e. the probability distribution of the number of links of each node) which followed a power law with exponent $\gamma=5.0$. The present work complements and extends our previous investigation of bone canal systems in terms of complex networks with respect to two particularly important issues frequently investigated in complex networks research, namely modularity (or community structure) and resilience to attacks. In particular, we apply the method reported by A. Clauset et al. ${ }^{16}$ in order to search for modular organization in a whole bone network extracted from the cortical structure of a whole chicken phalange. The presence of communities in a biological network provides valuable information about its mesoscopic organization, since communities are often closely related to the functionality and development of the respective networks. We also investigate the resilience of the bone network with respect to two different types of attacks, namely random failure process (RFP) - which simulates random canal breakdown along the network such as those induced by osteoporosis, as well as topological failure process (TFP)which simulates the behaviour of the bone canals structure with respect to progressive failures such as those implied by neoplasies and infections. The term attack has been used in the complex networks literature in order to express unwanted modifications in the original topology of a given network. Typically, one is interested in quantifying the progressive disruption of different types of attacks on the structure of the investigated networks, such as by monitoring a given set of features (e.g. maximum flow or size of the network). In the present work, the original bone network is artificially altered according to two different types of attacks in order to simulate biologically relevant processes such as distributed or localized injuries and diseases.

This paper is organized as follows. First, we present some of the principal biological concepts in bone formation and maintenance, as well as the procedures through which the bone network investigated in this work has been obtained. After describing the main concepts and methods for network analysis, the results regarding the modularity and resilience of the bone canals structure are described and discussed.

\section{Biological concepts}

The bone canal network is continuously broken down by osteoclasts and rebuilt by osteoblasts in a process called remodelling which is regulated by genetic, biological, and biomechanical factors ${ }^{17}$. At birth, cortical bone consists largely of woven bone with vascular spaces lined with osteoblasts. The osteoblasts reduce the volume of the vascular spaces by deposition of successive layers of new bone. The areas that remain are called primary osteons. These osteons are often, but not always, parallel to the long bone axis and may contain one to several vascular canals. In the adult cortical bone, new canals are created by osteoclasts, which excavate through the bone to create a "cutting cone", followed by a closing cone of osteoblasts that fill the canal with new bone $^{18}$ until the diameter of the cavity is reduced to a small, singular vascular canal. The newly formed bone cylinders are called secondary osteons or Haversian systems. It has been observed that the orientation of osteons is predominantly parallel to the marrow canal, which relates to the local primary loading direction. ${ }^{19}$ The Haversian canals are arranged in parallel to the marrow canal, and their diameters vary from 20 to $150 \mu \mathrm{m}$. This variation depends on the canal age, position in bone and the animal species. ${ }^{20}$ The Volkman's canals are similarly organized, but perpendicularly to the marrow canal. The shapes of the Haversian and Volkman's canals are almost cylindrical. ${ }^{3}$

During growth, load increases gradually, leading to a change in bone density and architecture. ${ }^{21,22}$ In cortical bone, it is particularly important that such a new structure be able to withstand the increasing bending load. Bending strength can be enhanced by enlarging the circumference of the bone, thereby increasing the cross-sectional moment of inertia. This expansion involves newly formed bone at the periosteal surface, in which blood vessels can be entrapped. Of the resultant canals, primary osteons are formed which are filled with bone by osteoblasts. If growth occurs rapidly, it may lead to a highly porous bone structure due to the rapid formation of primary osteons at the periosteal surface and the incomplete filling by the osteoblasts. Therefore, the organization of the bone canals system is intrisically related to molecular processes required for the signalling, development, and maintenance of the cellular framework within whole bones.

Despite the great importance of such canals for vascularization, mechanical properties, development and molecular processes, relatively few works have focused the comprehensive characterization and analysis of their connectivity and structure. Cohen and Harris ${ }^{23}$ studied this structure from 3D reconstruction of histological sections and more recently, advanced techniques of computational tomography have been proposed $^{2,24}$ in order to help the study of bone structure.

\section{The acquisition of the bone network}

In this article we investigate a complex network representing the canals structure of a cortical bone, corresponding to the whole phalange from the right leg of a young chicken. This bone was submitted to classical histological procedure resulting in about 800 two-dimensional slices. The 3D reconstruction of the bone canals and respective representation as a network was obtained by using a methodology similar to that described in a previous paper ${ }^{15}$, but with a higher level of automation as well as a smaller degree operator intervention. Initially, the slices were assembled in order to build the respective $3 \mathrm{D}$ volume. The $\mathrm{VTK}^{25}$ class library was used to visualize this volume and process it. Each canal was tracked and the intersections between canals were represented as nodes of a complex network, while the canals yielded the edges. A similar mapping procedure was also used by J. Buhl et al. ${ }^{26}$ in order to study the galleries built by ants and by $\mathrm{S}$. Laemmer $e t$ al. $^{27}$ as the means to investigate the topology of urban roads.

\section{Communities}

Many real-world networks present a modular structure, where sets of nodes concentrate many edges while being weakly connected one another. Generally, such a set is called a 
community. Some authors have suggested ${ }^{28-31}$ that each community has a specific function within a network and that its respective properties can differ strongly from the properties of the entire network. The detection of communities in complex network has been intensively explored along the last years and many algorithms have been developed for this purpose, such as spectral, ${ }^{32,33}$ hierarchical $^{13,34}$ and modularity based ${ }^{35,36}$ methods. Here, we applied the algorithm presented by Clauset et $a l .{ }^{16}$ in order to identify the communities in the bone network. This algorithm is based on the maximization of the modularity $(Q)$ measurement, which quantifies the degree of separation between the communities. Generally, a modularity value above 0.3 is understood as a strong indication of modularity in the network. ${ }^{37}$

\section{Network resilience to failures}

One particularly important feature of the connectivity of specific types of networks regards their respective resilience to attacks and failures. ${ }^{38,39}$ For instance, how long can a given network undergoing random loss of edges keep most of its nodes connected? In the case of bone networks, resilience is required with respect to a certain degree of failures implied by obstruction of channels occurring as a consequence of diseases or injuries. One way to quantify the resilience of a network to failure consists in monitoring the size of its giant connected component $^{40}$ (i.e. the largest set of connected nodes in the graph, in the sense of each of these nodes being reachable from any other node in the component through at least one path) while the network is undergoing degradation. The results reported in the literature ${ }^{38,39}$ show that networks tend to present a giant component bellow a critical fraction $p_{\mathrm{c}}$ of deleted edges. Above this value, the network is fragmented and its functionality is undermined. Cascade failure is another type of degradation of networks related, now with respect to the edge capacity. When the flow through an edge is higher than its capacity, the edge is lost and the flow is redirected to the neighbouring edges. However, such neighbouring edges can be overloaded too, so that the process can propagate continuously through all edges up to the network final breakdown. The size of the giant component ${ }^{40}$ and the global efficiency $E,{ }^{41}$ defined as the inverse of the path length between the node $i$ and $j$ averaged over all pairs $(i, j)$, have been used in order to evaluate the damage of cascade failure.

The effect of modular structure in cascade failure processes was recently investigated by Jiang-jun $\mathrm{Wu}$ et $a l^{42}$ Those authors showed that global efficiency of higher modular networks tends to be larger than networks with small modularity values during the cascade process. Moreover, the average shortest path length is smaller in the higher modular networks. These findings indicate that community organization tends to improve the performance of networks in presence of failures. Here, we considered two types of failures. The first, called random failure process (RFP) is similar to random breakdown ${ }^{38-40}$ and can be used to simulate random canal breakdown along the network, as shown in Fig. 1B. The RFP type of failure can also be used to model the effects of osteoporosis, which degrades calcium in the canals at random places along the bone. The second type of failure considered in
(A)

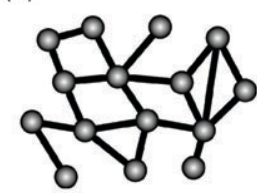

(B)

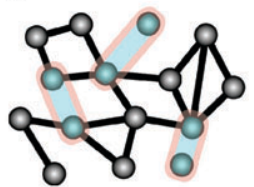

(C)

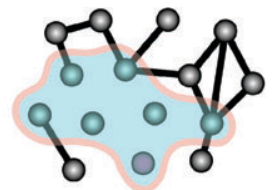

Fig. 1 Types of failures considered in the current paper. (A) Sample of a generic network. (B) Random failure process (RFP): edges are randomly selected and deleted from the network. (C) Topological failure process (TFP): starting from a seed node, all edges are deleted until the network extremity is reached (the seed node is shown in purple). Note that RFP can be used to simulate osteoporosis, while TFP is analogous to lesions along the bone structure. The deleted edges are shown within the blue region.

the current work is the topological failure process (TFP), similar to cascade failure but without considering weights, in which the damage spreads from a specific seed (i.e. a node) through its successive neighbours. From such a seed, the failure spreads and deletes all neighbouring edges until the network border is reached, as shown in Fig. 1C. The TFP simulates the effects of a lesion, where all canals at specific places are damaged, as well as diseases which progress through adjacent canals, such as neoplasies and infections.

\section{Random bone network and regular bone network}

In order to compare the robustness of the bone network with a null reference model, we considered a randomized version of the bone network, obtained by using the algorithm proposed by S. Maslov and K. Sneppen, ${ }^{43}$ where pairs of edges are randomly selected and their extremities swapped. The random version of the bone network generated by this method retains the degree distribution, but looses all correlation among the node degrees. We also generated a regular bone network, i.e. with the same average degree and the same number of nodes as the original bone network. In order to do so, we placed nodes on randomly chosen positions of a three-dimensional grid and added the corresponding number of edges between neighbours. Such a procedure was performed so as to obtain only one connected component.

The robustness of the bone network and its random and regular versions against both RFP and TFP attacks was investigated through two measures: the size of giant component and the maximum flow, described as follows:

(i) Size of giant component $(S)$ : Corresponds to the number of nodes present in the largest component of the network. This measurement is important because, as the bone network should be able to convey nourishment to all bone cells, the size of the giant connected component should be as large as possible.

(ii) Maximum flow $(\Phi)$ : Another important feature related to transportation in networks is the maximum flow. ${ }^{44}$ In transportation networks, the edges have maximum transportation capacity, which can be related to the diameter and length of the canals diameter, as well as the physical properties of the materials used in the network. Thus, it is natural to ask what is the maximum flow that can circulate from source to sink in a particular network. Here we consider that all edges 
have unitary capacity. In order to evaluate the transportation robustness of the bone network, the maximum flow was monitored during the attack dynamics.

\section{Results and discussion}

In Fig. 2A we show the bone network obtained by using the methodology described above. It contains 5592 nodes and average degree $\langle k\rangle=2.37$. Fig. $2 \mathrm{~B}, \mathrm{C}$ and $\mathrm{D}$ show some of the main topologic features of the obtained bone network, including its degree distribution, shortest path length distribution and degree-degree correlation matrix. We can see that, as in the previous paper, ${ }^{15}$ this bone network also follows a degree power-law with exponent $\gamma=6.0$ and cut-off $k_{\mathrm{c}}=10$, which is a consequence of the spatial restriction of the system. Another implication of this restriction is the absence of the small-world phenomena (i.e. a small value of average shortest path lengths between pairs of nodes), as we can observe in Fig. $2 \mathrm{C}$, where the average shortest path length is close to $\langle L\rangle=30$. In addition, Fig. 2C shows that the most common connection in this network occurs between nodes with degree $k=k^{\prime}=3$. Since the same result was obtained for the cat femur network, ${ }^{15}$ it suggests a possible universal feature of bone canals system.
The application of Clauset et al.'s algorithm ${ }^{16}$ led to a modularity index $Q=0.85$, indicating that the bone network is highly modular. At this value, the network is partitioned into 15 communities, which are shown in Fig. 3 (geographical and topological views). We observed that the communities located at the posterior portion of the bone tend to be denser, such as the communities 1 and 2 (respectively red and yellow in Fig. 2A), with respective average degrees $\langle k\rangle_{1}=\langle k\rangle_{2}=2.75$. On the other hand, the communities at the anterior portion are similar to trees, involving fewer cycles. In order to better characterize the differences among the bone communities, we obtained relevant measurements (namely $\langle k\rangle$-average degree, $\langle C C\rangle$-average clustering coefficient, $\langle L\rangle$-average shortest path length and $L_{\max }$-diameter or maximum value of the shortest path length) from each community and used the PCA transformation $^{45}$ in order to visualize the results. The PCA transformation can be applied in order to project the data into a two-dimensional space with axis given by linear combinations of original measurements. In this way, we can observe the emergence of clusters of communities which share common features. Fig. 4 illustrates the results obtained by PCA, where we can identify two main clusters. The smallest of them contains the most dense communities, all of which located at

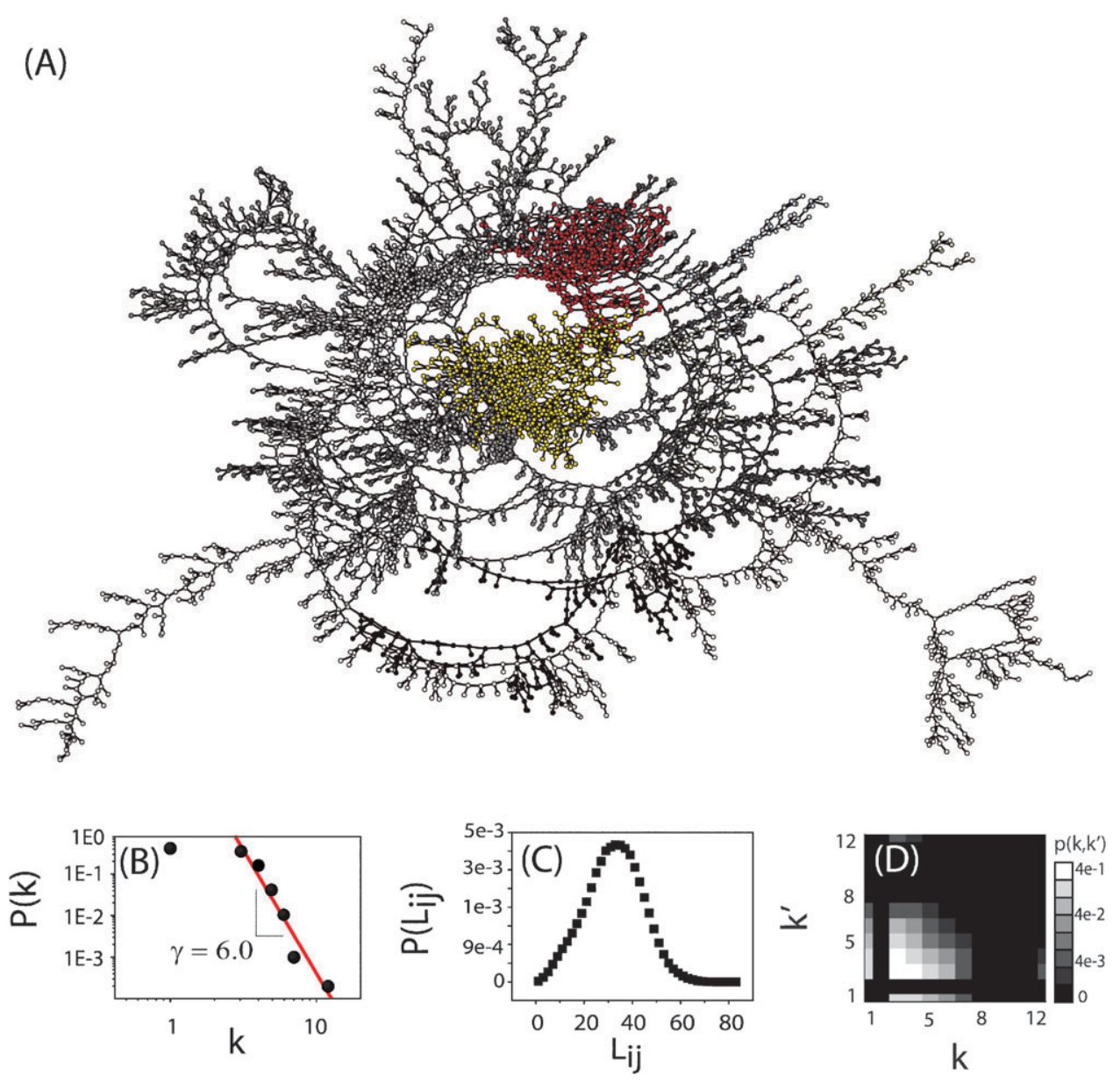

Fig. 2 Main Features of the Bone Network. (A) The bone network investigated in this article contains 5592 nodes and average degree $\langle k\rangle=2.375$. Each gray level corresponds to a detected community. The most connected communities are marked in red and yellow, identified as $1\left(\langle k\rangle_{1}=2.75\right)$ and $2\left(\langle k\rangle_{2}=2.75\right)$, respectively. (B) Degree distribution of the bone network. (C) Distribution of shortest path length $L$, and (D) degree-degree correlation matrix. 

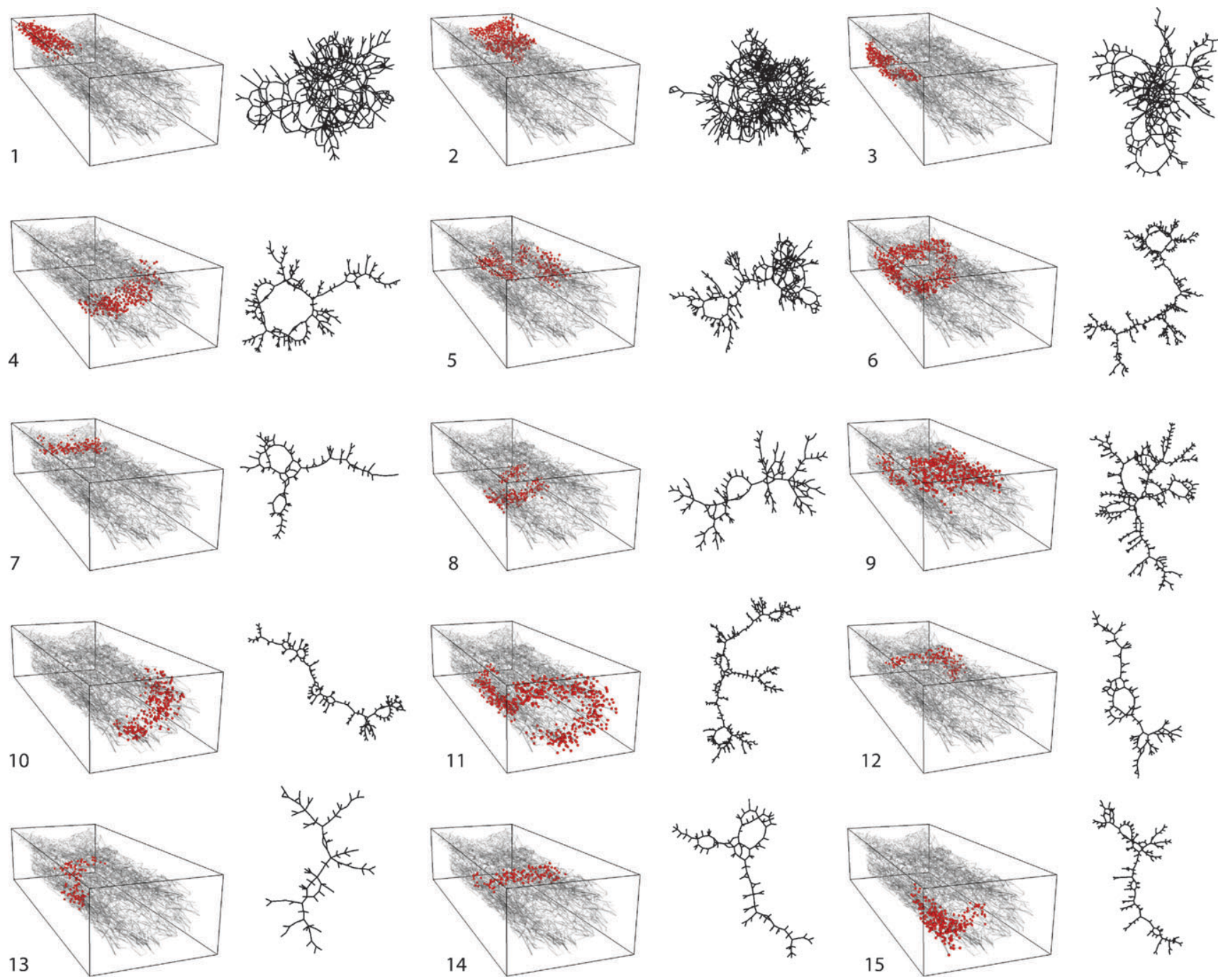

Fig. 3 Modular organization of the bone network. Spatial location of each community in the cortical bone structure. Note that the communities 1 and 2 are located in the posterior region of the bone. Also interesting is the increase of community complexity from the posterior to the anterior parts. For example, the extreme anterior communities, labelled as 10 and 15 , have average degrees $\langle k\rangle_{10}=2.19$ and $\langle k\rangle_{15}=2.12$.

the posterior region of the bone. That region also includes two protrusions which are particularly important for the mechanical properties of the bone. It is possible that the stronger pressures and tensions exerted over these protrusions could be responsible for the increased connectivity of the communities. It is also possible that the differences in density of connections observed along the posterior-anterior axis be a consequence of different stages of development, in the sense that the provisionally simple communities at the anterior region may become denser later.

In order to investigate the resilience of the bone network against failures, as well as the respective influence of the modular structure, the following simulations were performed. The size of the giant connected component $(S)$ and the maximum flow $(\Phi)$ were monitored while the bone network and its randomized and regular version were submitted to RFP and TFP attacks. Fig. 5A shows the damage caused by RFP on the size of the giant component. The result indicates that for removals of $35 \%$ or less of the edges, the robustness of the bone network is intermediate to those of the regular and random versions.

After $45 \%$ or more of the edges have been removed, the behaviour of the bone network becomes almost identical to that of the respective random bone network, while the regular bone network allows a better resilience. This result suggests that the modular bone network is optimized in order to survive to low injury levels, but not as much as the resilience allowed by long range connections present in the random bone network. It may also explain the fact that advanced stages of several bone diseases, such as osteoporosis, can be so catastrophic. Under the TFP (Fig. 5B) and for percentages of removed edges smaller than $35 \%$, the bone network shows a performance similar to the respective randomized version and better than regular version. This result suggests that the modular structure of the bone network can be a possible means to compensate for the lack of long range connections that are present in random version. Above this value, the resilience of the bone network becomes the worst. 


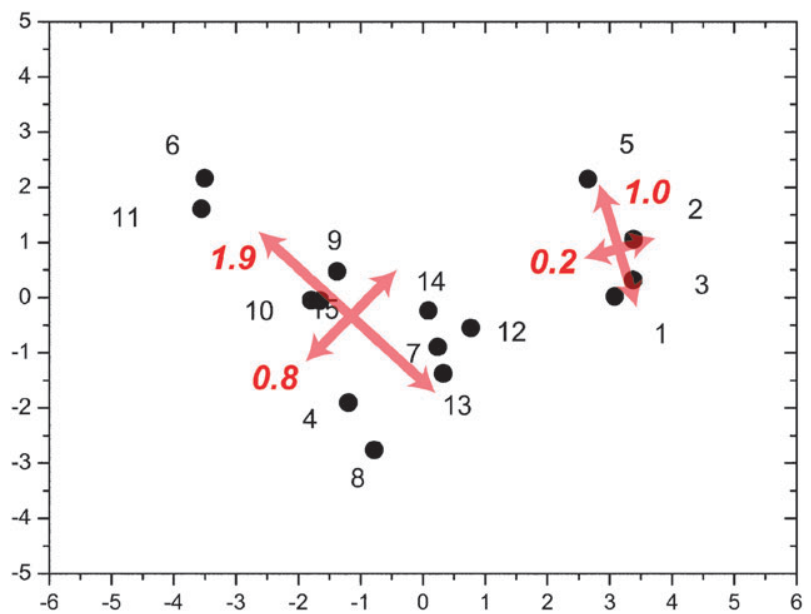

Fig. 4 PCA of the communities features. Principal component analysis was performed considering the following measurements: $\langle k\rangle$ average degree, $\langle C C\rangle$-average clustering coefficient, $\langle L\rangle$-average shortest path and $L_{\max }$-diameter (maximum shortest path). Two main clusters are clearly identified. All communities in the smallest cluster are located at the posterior portion of the bone. The PCA axes obtained for each cluster, as well as the respective variances, are also shown in this figure.
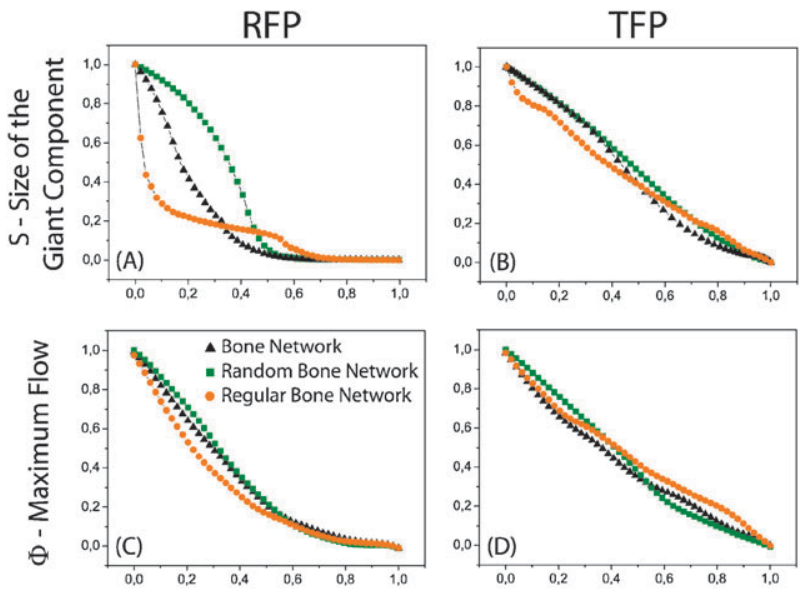

Fraction of Removed Edges

Fig. 5 The robustness of bone network. Size of the giant connected component under (A) RFP and (B) TFP. Maximum flow in network under (C) RFP and D) TFP. Three networks are considered: bone network, randomized bone network and regular bone network. The curves, which represent the averages obtained for 500 realizations, were normalized by the maximum value in each network.

Fig. 5C and D present the results of failure over the maximum flow in the bone network. These results indicate that the modular structure of the bone has little influence over the transportation dynamics. It is important to observe that these results refer to unweighted networks. It is possible that a more definite effect of the modularity on resilience be observed for versions of the bone network where the weights correspond to the diameter of the canals. However, such effects can be expected to be limited as the diameters are almost uniform in the case of the investigated bone.

\section{Conclusions}

Several biological systems are characterized by intricate patterns of connectivity, which are the result of long evolutionary processes aimed at optimizing specific functionalities. In this article we presented how the three-dimensional reconstruction of the canals structure of a whole bone, followed by respective representation in terms of a complex network, allowed the identification and quantification of important structural patterns of organization of the canals connectivity. In agreement with our previous paper, ${ }^{15}$ the degree distribution was found to follow a power law with cut-off. In addition, because the connectivity is largely constrained by spatial adjacency between the canals, no small-world effect was verified. At the same time, the bone canals network was found to be highly modular, including several communities with distinct connectivity patterns. The application of principal component analysis allowed the identification of two clear clusters of communities, differing in density of connections and position along the main axis of the bone. Interestingly, the communities at and near the bone protrusions were found to be the most dense in connections, which is possibly related to the special mechanical function of those regions, requiring enhanced vascularization and being subjected to higher mechanical stress. The identification of communities in the bone canals structure is also closely related to several molecular issues in development, abnormalities and diseases of bones. It would be particularly interesting to investigate to which extent the bone modular structure is defined and controlled by molecular mechanisms, while also reflecting environmental effects such as mechanical stimuli. In addition, being immediately related to cell-to-cell signalling within the bone, the topology of the system of canals is continuously affected by molecular signalling during ontogenesis and even along more mature stages. Indeed, the identification of progressively more connected communities along the anterior-posterior axis of the bone seems to indicate the presence of transcription factor gradients. Therefore, the comprehensive quantification of the topological properties of the bone canals reported in this work paves the way for investigations of how eventual changes (e.g. mutations) in the respective molecular programmes affect the bone structure and maintenance, particularly with respect to the BMP (bone morphogenic proteins) ${ }^{46-48}$ family of genes. The comprehensive characterization of the bone canals connectivity is also critical for better understanding how molecular changes and malfunctions can lead to abnormal development and diseases (e.g. osteoporosis).

In order to complement the characterization of the topological and modular analysis of the bone network, we also performed simulations of different types of network failures, more specifically random attacks and cascade of failures. The original bone network was compared to its randomized version as well as to an equivalent regular structure. The modular structure of the bone network does not seem to have any specific influence on the behaviour of the transportation dynamics from the perspective of maximum flow. However, the bone network was found to be more robust than its regular version and less robust than its random version with respect to random attacks, which simulate diseases such as osteoporosis. 
Such a systematic investigation of the resilience of the bone canals system to different types of attacks is also related to several molecular issues. For instance, networks of flow sensors and molecular signalling are possibly required in order to guarantee proper maintenance of the canal topology so as to ensure adequate mechanical properties, flow of nutrients and removal of unwanted materials.

Several are the possibilities for future related research, which include the investigation of the bone structure along several developmental stages, comparison between bone canal systems from different animals, as well as the study of the relationship between molecular issues (e.g. specific gene families and pathological processes) and the respective phenotypic properties of canals systems.

\section{Acknowledgements}

Luciano da F. Costa thanks Human Frontier Science Program RGP39/2002, FAPESP (procs. 99/12765-2 and 05/00587-5) and $\mathrm{CNPq}$ (proc. 301303/06-1) for financial support. Matheus P. Viana thanks FAPESP (proc. 07/50882-9). Marcelo E. Beletti thanks CNPq (proc. 131903/05-5 and 350058/03-2) for financial support and Esther Tanck thanks NWO-STW (NPG.6778) for financial support.

\section{References}

1 S. C. Cowin, in Bone Mechanics Handbook, 2nd edn., 2001, Informa Health Care, vol. 1, ch. 4, pp. 21.1-26.1.

2 D. M. L. Cooper, A. L. Turinsky, C. W. Sensen and B. Hallgrímsson, Anat. Rec., 2003, 274B, 169-179.

3 F. C. McLean and M. R. Irist, in Fundamentals of the Physiology of Skeletal Tissue, The University of Chicago Press, 3nd edn., 1968, vol. 1.

4 R. Albert and A.-L. Barabási, Rev. Mod. Phys., 2002, 74, 47-97.

5 S. N. Dorogovtsev and J. F. F. Mendes, Adv. Phys., 2002, 51, 1079-1187.

6 M. E. J. Newman, SIAM Review, 2003, 45, 167-256.

7 L. A. N. Amaral and J. M. Ottino, Eur. Phys. J. B, 2004, 38, $147-162$.

8 L. da F. Costa, F. A. Rodrigues, G. Travieso and P. R. Villas Boas, Adv. Phys., 2007, 56, 167-242.

9 A. Vázquez, A. Flammini, A. Maritan and A. Vespignani, Complexus, 2003, 1, 38-44.

10 G. Bagler and S. Sinha, Physica A, 2005, 346, 27-33.

11 H. Jeong, B. Tombor, R. Albert, Z. N. Oltvai and A.-L. Barabási, Nature, 2000, 407, 651-654.

12 E. Ravasz, A. L. Somera, D. A. Mongru, Z. Oltvai and A.-L. Barabási, Science, 2002, 297, 1551-1555.

13 L. da F. Costa, Proceedings of the Coevolution and self-organanization in dynamical networks meeting, Salou, 2005.

14 L. da F. Costa and L. Diambra, Phys. Rev. E, 2005, 71, 021901-021907.

15 L. da F. Costa, M. P. Viana and M. E. Beletti, Appl. Phys. Lett., 2006, 88, 033903-033905.
16 A. Clauset, M. E. J. Newman and C. Moore, Phys. Rev. E, 2004, 70, 066111-066117.

17 D. R. Carter, M. Wong and T. E. Orr, J. Biomechanics, 1991, 24, 3-16.

18 A. M. Parfitt, J. Cell. Biochem., 1994, 55, 273-286.

19 M. Petrtyl, J. Hert and P. Fiala, J. Biomech., 1996, 29, 161-169.

20 R. Georgia and I. Albu, Embryol., 1988, 34, 155-159.

21 E. Tanck, J. Homminga, G. H. van Lenthe and R. Huiskes, Bone, 2001, 28, 650-654.

22 E. Tanck, G. Hannink, R. Ruimerman, P. Buma, E. H. Burger and R. Huiskes, J. Anat., 2006, 208, 73-79.

23 J. Cohen and W. Harris, J. Bone Jt. Surg., Am. Vol., 1958, 40, 419-434.

24 B. R. McCreadie, R. W. Goulet, L. A. Feldkamp and S. A. Goldstein, Adv. Exp. Med. Biol., 2001, 496, 67-83.

25 W. J. Schroeder, L. S. Avila and W. Hoffman, IEEE Comput. Graphics Appl., 2000, 20, 20-27.

26 J. Buhl, J. Gautrais, R. V. Sol, P. Kuntz, S. Valverde, J. L. Deneubourg and G. Theraulaz, Eur. Phys. J. B, 2004, 42, 123-129.

27 S. Laemmer, B. Gehlsen and D. Helbing, Phys. A, 2006, 363, 89-95.

28 G. W. Flake, S. R. Lawrence, C. L. Giles and F. M. Coetzee, IEEE Computer, 2002, 35, 66-71.

29 P. Holme, M. Huss and H. Jeong, Bioinformatics, 2003, 19, $532-538$.

30 R. Guimerá and L. A. N. Amaral, Nature, 2005, 433, 895-900.

31 M. E. J. Newman, Proc. Natl. Acad. Sci. U. S. A., 2006, 103, $8577-8582$.

32 M. Fiedler, Czech. Math. J., 1970, 23, 298-305.

33 A. Pothen, H. Simon and K.-P. Liou, SIAM J. Matrix Anal. Appl., 1990, 11, 430-452.

34 J. Scott, in Social Network Analysis: A Handbook, Sage Publications Ltd, 2nd edn, 2000, vol. 1, ch. 7, pp. 123-145.

35 M. E. J. Newman and M. Girvan, Phys. Rev. E, 2004, 69, 026113-026128.

36 M. E. J. Newman, Phys. Rev. E, 2004, 69, 066133-066137.

37 M. E. J. Newman, Phys. Rev. E, 2006, 74, 036104-036123.

38 R. Cohen, K. Erez, D. ben Avraham and S. Havlin, Phys. Rev. Lett., 2000, 85, 4626-4628.

39 B. Wanga, H. Tanga, C. Guoa, Z. Xiub and T. Zhouc, Physica A, 2006, 368, 607-614.

40 P. Crucitti, V. Latora, M. Marchiori and A. Rapisarda, Physica A, 2003, 320, 622-642.

41 A. E. Motter, T. Nishikawa and Y.-C. Lai, Phys. Rev. E, 2002, 66, 065103-065106.

42 J.-j. Wu, Z.-y. Gao and H. J. Sun, Phys. Rev. E, 2006, 74, $066111-066115$

43 S. Maslov and K. Sneppen, Science, 2002, 296, 910-913.

44 G. Hadley, in Linear Programming, Addison-Wesley Pub. Co., 1st edn, 1962, vol. 1, ch. 10, pp. 331-378.

45 L. da F. Costa and R. M. Cesar, Jr, in Shape Analysis and Classification: Theory and Practice, CRC Press, 1st edn., 2001, vol. 1 , ch. 8, pp. 560-564.

46 J. M. Wozney, V. Rosen, A. J. Celeste, L. M. Mitsock, M. J. Whitters, R. W. Kriz, R. M. Hewick and E. A. Wang, Science, 1989, 242, 1528-1534.

47 B. L. Hogan, Genes Dev., 1996, 10, 1580-1594.

48 E. M. Shore, M. Xu, P. B. Shah, H. B. Janoff, G. V. Hahn, M. A. Deardorff, L. Sovinsky, N. B. Spinner, M. A. Zasloff, J. M. Wozney and F. S. Kaplan, Calcif. Tissue Int., 1998, 63, 221-229. 